\title{
Structural variants are a major source of gene expression differences in humans and often affect multiple nearby genes
}

\author{
Alexandra J. Scott, ${ }^{1,2}$ Colby Chiang, ${ }^{1,2}$ and Ira M. Hall ${ }^{1,2,3}$ \\ ${ }^{1}$ McDonnell Genome Institute, Washington University School of Medicine, St. Louis, Missouri 63108, USA; ${ }^{2}$ Department of Medicine, \\ Washington University School of Medicine, St. Louis, Missouri 63110, USA; ${ }^{3}$ Department of Genetics, Yale University School of \\ Medicine, New Haven, Connecticut 06510, USA
}

\begin{abstract}
Structural variants (SVs) are an important source of human genome diversity, but their functional effects are poorly understood. We mapped 61,668 SVs in 613 individuals from the GTEx project and measured their effects on gene expression. We estimate that common SVs are causal at $2.66 \%$ of eQTLs, a 10.5 -fold enrichment relative to their abundance in the genome. Duplications and deletions were the most impactful variant types, whereas the contribution of mobile element insertions was small ( $0.12 \%$ of eQTLs, 1.9 -fold enriched). Multitissue analysis of eQTLs revealed that gene-altering SVs show more constitutive effects than other variant types, with $62.09 \%$ of coding SV-eQTLs active in all tissues with eQTL activity compared with $23.08 \%$ of coding SNV- and indel-eQTLs. Noncoding SVs, SNVs and indels show broadly similar patterns. We also identified 539 rare SVs associated with nearby gene expression outliers. Of these, $62.34 \%$ are noncoding SVs that affect gene expression but have modest enrichment at regulatory elements, showing that rare noncoding SVs are a major source of gene expression differences but remain difficult to predict from current annotations. Both common and rare SVs often affect the expression of multiple genes: SV-eQTLs affect an average of 1.82 nearby genes, whereas SNV- and indeleQTLs affect an average of 1.09 genes, and $21.34 \%$ of rare expression-altering SVs show effects on two to nine different genes. We also observe significant effects on rare gene expression changes extending $1 \mathrm{Mb}$ from the SV. This provides a mechanism by which individual SVs may have strong or pleiotropic effects on phenotypic variation.
\end{abstract}

[Supplemental material is available for this article.]

Structural variants (SVs) are a diverse class of genetic variation that include copy number variants (CNVs), mobile element insertions (MEIs), and balanced rearrangements at least $50 \mathrm{bp}$ in length. Although SVs are relatively rare compared with single-nucleotide variants (SNVs) and small insertion or deletion (indel) variants, their size and diversity mean that SVs can disrupt protein-coding genes and genomic regulatory elements through diverse mechanisms. Furthermore, SVs often have more severe consequences compared with smaller variants, and previous studies have found that SVs have an outsized impact on human gene expression compared with their relative abundance in the genome (Stranger et al. 2007; Sudmant et al. 2015; Chiang et al. 2017). SVs have also been implicated in the biology of human diseases such as autism spectrum disorder (Sebat et al. 2007; Weiss et al. 2008; Turner et al. 2017; Brandler et al. 2018) and schizophrenia (International Schizophrenia Consortium 2008; Walsh et al. 2008; McCarthy et al. 2009; Marshall et al. 2017). However, SVs are difficult to detect from short-read DNA sequencing data and are often excluded from complex trait association studies.

Advances in high-throughput sequencing technologies that have allowed for widespread use of whole-genome sequencing (WGS), combined with advances in scaling SV detection algorithms, mean that comprehensive studies of all forms of genetic variation are now possible for large human cohorts. Recent studies of SV in large, deeply sequenced human cohorts have found that

Corresponding author: ira.hall@yale.edu

Article published online before print. Article, supplemental material, and publication date are at https://www.genome.org/cgi/doi/10.1101/gr.275488.121. Freely available online through the Genome Research Open Access option.
SVs account for $4.0 \%-11.2 \%$ of rare high-impact coding alleles (Abel et al. 2020) and are responsible for $25 \%-29 \%$ of rare protein-truncating events per genome (Collins et al. 2020). However, few studies to date have examined the functional effects of SV on gene expression, and these studies are limited to relatively small cohort sizes or only a few tissue types with available gene expression data (Sudmant et al. 2015; Chiang et al. 2017; Han et al. 2020; Jakubosky et al. 2020).

Here, we use deep WGS data and multitissue RNA-seq expression data from 613 individuals in the Genotype-Tissue Expression (GTEx) project to comprehensively map SVs and to evaluate their impact on both common and rare gene expression changes in up to 48 tissue types (Supplemental Table S1). This study expands on our prior analysis of SV in 147 human samples from the GTEx cohort with RNA-seq expression data from 13 different tissues (Chiang et al. 2017) and is the most comprehensive study of SVeQTLs to date. The expanded cohort size provides greater power to evaluate the impact and mechanisms of SV-associated gene expression changes, particularly for rare SVs.

\section{Results}

\section{Variant calling}

We mapped SVs in 613 individuals from the GTEx v7 release using LUMPY (Layer et al. 2014; Chiang et al. 2015), svtools (Larson et al. 2019), GenomeSTRiP (Handsaker et al. 2011, 2015), and the

(C) 2021 Scott et al. This article, published in Genome Research, is available under a Creative Commons License (Attribution 4.0 International), as described at http://creativecommons.org/licenses/by/4.0/. 
Mobile Element Locator Tool (MELT; see Methods) (Gardner et al. 2017). Variant calls were filtered and merged using the same approach as in our previous GTEx study (Chiang et al. 2017; Li et al. 2017), resulting in a total of 61,668 "high-confidence" SVs that are the basis for all subsequent analyses (Table 1). SNVs and small insertion deletion (indel) variants were mapped using GATK (McKenna et al. 2010) as part of the official v7 release from the GTEx Consortium.

\section{Effects of common SVs}

We performed cis-eQTL mapping of common variants $(\mathrm{MAF} \geq$ 0.01) using a permutation-based mapping approach with FastQTL (Ongen et al. 2016), limiting comparisons to variants within $1 \mathrm{Mb}$ of the transcription start site (TSS) of each gene. We performed eQTL analyses in each of the 48 tissues for which expression data were available for at least 70 individuals (Supplemental Table S1), and defined an eQTL as an eVariant/ eGene pair detected in a given tissue. We performed a "joint" eQTL mapping analysis in which SVs, SNVs, and indels were simultaneously queried for eQTL status, allowing for direct comparisons between their properties and identification of a likely causal variant. An SV was the lead marker in 2.66\% (7960/ 299,187 ) of eQTLs (Supplemental Table S2), although this is likely an underestimate of SV causality owing to inferior genotyping accuracy for SVs, which biases eQTL fine-mapping analyses against SVs. Although this estimate of the contribution of SVs is relatively small, it represents a 10.5-fold enrichment over the abundance of SVs in the genome. This result is consistent with our prior analysis of the initial 147 individuals from the GTEx cohort (Chiang et al. 2017). In the same 13 tissues evaluated in this previous study, the increased sample size used here allowed us to identify 617 genes with SV-eQTLs that were not identified in the smaller study, although 57 genes from the initial study are no longer SV-eQTLs. As expected, many eSVs are large, although we observe smaller eSVs as well (Fig. 1A). Furthermore, 71.82\% (5717/7960) of all SV-eQTLs identified in this study are noncoding (Supplemental Fig. S1), meaning the SV does not intersect with any exons of its associated eGene. This figure is even more striking when eQTLs are collapsed across tissues, where 1907/2318 (82.27\%) of unique eGene/eSV pairs are noncoding. This also suggests that coding SV-eQTLs are more constitutive as more of them are identified in multiple tissues.
A novel aspect of this study is that we used MELT to sensitively map MEI variants, including nonreference insertions that were not detected in our prior GTEx study. It has been proposed that MEIs may have broad effects on gene expression owing to their ability to disrupt genes, promote epigenetic gene silencing, and serve as alternate promoters (Chuong et al. 2017; Payer and Burns 2019); however, there has been scant data in humans to address these hypotheses. We found that only $0.12 \%(353 / 299,187)$ of eQTLs had an MEI as the lead marker. Although this is a 1.9-fold enrichment of predicted causal MEIs relative to their abundance ( $0.06 \%$ of common variants), MEIs were far less likely than other SV types to be the lead marker (e.g., mCNVs are enriched 45fold, duplications 38-fold, and deletions 3.3-fold). It is unlikely that this relative depletion results simply from the small size of MEIs, as a size-matched analysis of MEIs and LUMPY deletions showed that only $2.74 \%$ of MEIs are eQTLs compared with $3.5 \%$ of deletions. When including LUMPY deletions of all sizes, $4.8 \%$ of deletions are eQTLs. This result is also unlikely to be explained by poor sensitivity or genotyping accuracy at MEIs considering that we detected slightly more MEIs per genome than a recent comprehensive long-read read study (Ebert et al. 2021) —with a mean of 1961 MEIs per genome versus 1637-and the linkage disequilibrium (LD) patterns at MEIs relative to nearby SNVs mirror that of LUMPY deletions (Supplemental Note; Supplemental Fig. S2). Thus, despite compelling molecular evidence for the functional potential of MEIs, our results suggest that they are only slightly enriched as causal eQTL variants relative to SNVs and indels and are depleted relative to other SVs, on average.

We found that not only do SVs have larger effect sizes compared to SNPs and indels, as noted in previous studies (Supplemental Fig. S1; Jakubosky et al. 2020; Chiang et al. 2017), they are also more likely to alter the expression of multiple nearby genes. Each eSV affects an average of 1.82 unique eGenes, whereas SNVs and indels affect an average of 1.09 unique eGenes. Although this effect is partially explained by large SVs that alter the copy number of multiple adjacent genes, there is also a significant difference for genes affected by noncoding eVariants: On average, eSVs affect 1.50 unique eGenes for which they do not intersect any exons of the eGene compared with an average of 1.04 unique eGenes for SNVs and indels $\left(P=1.02 \times 10^{-55}\right.$, one-sided MannWhitney $U$ test) (Fig. 1B-D). These noncoding effects are most pronounced for duplications $\left(P=6.10 \times 10^{-53}\right)$ and mCNVs $(P=4.75 \times$ $\left.10^{-56}\right)$, which are the only two categories of noncoding SVs that

Table 1. Summary of variant types and eQTL mapping

\begin{tabular}{|c|c|c|c|c|c|}
\hline & Detection method & No. of variants & Median size (bp) & No. of common variants & eVariants \\
\hline SNV & GATK & $37,087,030$ & 1 & $9,609,545$ & 178,000 \\
\hline Indel & GATK & $3,081,270$ & 3 & 818,401 & 16,460 \\
\hline \multirow[t]{2}{*}{ Deletion (DEL) } & $\mathrm{BP}$ & 20,954 & 1311 & 4385 & 210 \\
\hline & RD & 10,252 & 2151 & 8166 & 66 \\
\hline \multirow[t]{2}{*}{ Duplication (DUP) } & $\mathrm{BP}$ & 3388 & 2632 & 1090 & 64 \\
\hline & RD & 1598 & 6891 & 896 & 233 \\
\hline Multiallelic CNV (mCNV) & $\mathrm{RD}$ & 4365 & 3602 & 3238 & 460 \\
\hline Inversion & $\mathrm{BP}$ & 295 & 1054 & 96 & 2 \\
\hline Reference mobile element insertion (MEl-del) & BP & 2681 & 306 & 2026 & 88 \\
\hline Nonreference mobile element insertion (MEl-ins) & $\mathrm{BP}$ & 13,066 & 280 & 4496 & 91 \\
\hline Other (BND) & BP & 5069 & - & 2010 & 57 \\
\hline All SVs & - & 61,668 & - & 26,409 & 1271 \\
\hline All variants & - & $40,229,968$ & - & $10,454,355$ & 195,731 \\
\hline
\end{tabular}

SVs were detected based on breakpoint evidence (BP) or read-depth evidence (RD). SNVs and indels were called using the Genome Analysis Toolkit (GATK). Common variants (MAF $\geq 0.01$ ) were used to map cis-eQTLs.

\section{Genome Research}

www.genome.org 
SVs have broad regional effects in humans
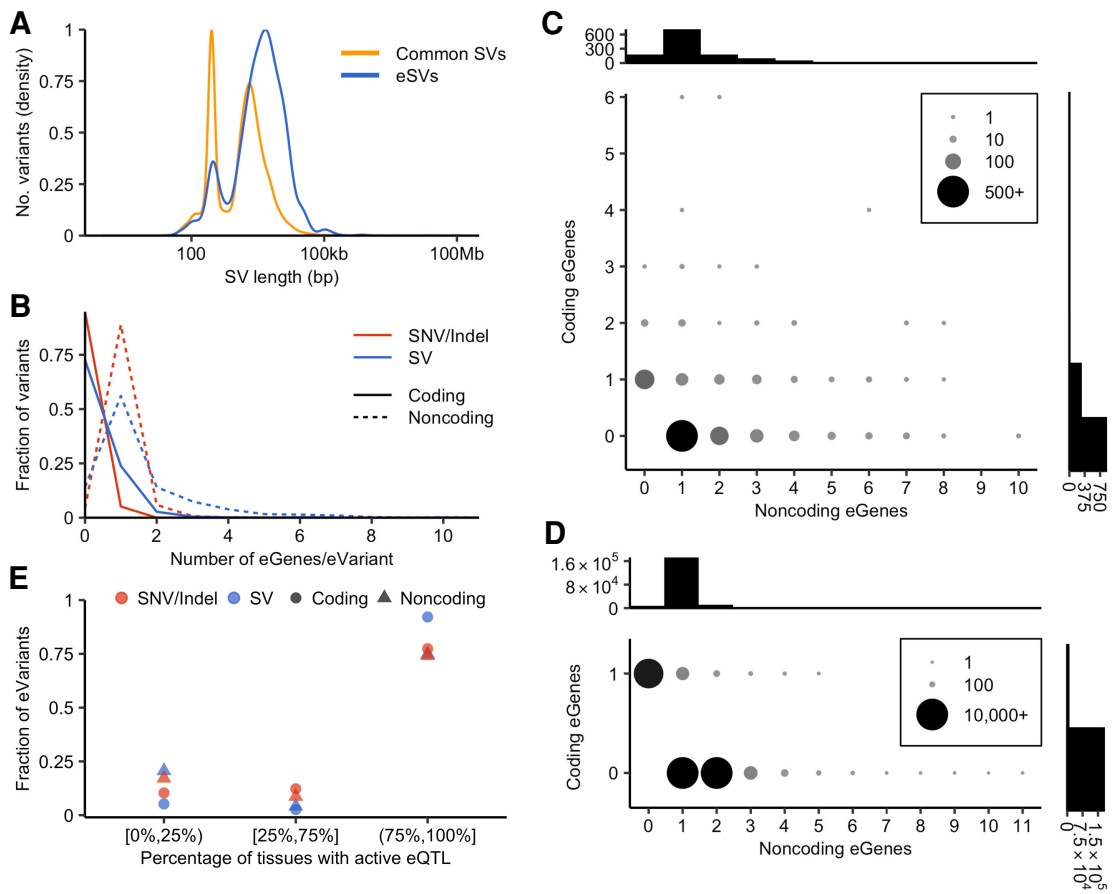

Figure 1. Features of SV-eQTLs. (A) Size distribution of eSVs compared with all common SVs. (B) Distribution of the number of eGenes per eVariant for SVs compared with SNVs and indels. "Coding" eGenes refer to eGenes whose exons are intersected by the associated eVariant, and "noncoding" eGenes are not intersected by the associated eVariant. Counts are shown for every eVariant; thus, eVariants with zero coding or zero noncoding eGenes are included in the distributions. $(C, D)$ The number of eVariants, as shown by dot size and color, with the indicated combination of coding and noncoding eGenes, as defined above. Shown for SVs $(C)$ and SNV/indels $(D)$, with histograms showing the total number of eVariants, with the indicated number of associated coding or noncoding eGenes above the $y$ - and $x$-axes, respectively. (E) Distribution of tissue specificity of eQTLs across tissues as evaluated by METASOFT, separated into the lowest quartile, middle two quartiles, and top quartile, for eQTLs in which the activity status is known in at least 43 of 48 evaluated tissues. The points indicate the fraction of SVeQTLs or SNV- and indel-eQTLs that are active $(m>0.9)$ in the proportion of tissues indicated on the $x$ axis.

affect significantly more eGenes than point variants. This result indicates that causal SVs are generally more impactful than causal point variants, in terms of both their per-gene effect sizes and their potential to affect multiple genes. These results also suggest that SVs are more likely to disrupt key regulatory elements and/or alter higher-order genome architecture, allowing individual variants to affect multiple genes.

To investigate the functional mechanisms of expression-altering SVs, we defined a set of putative causal SVs using a score generated by taking the product of the causal probability calculated using CAVIAR (Hormozdiari et al. 2014) and the fraction of heritability attributed to the SV calculated using GCTA (Supplemental Table S3; Yang et al. 2011), as described previously (Chiang et al. 2017). At each eGene, we selected the SV within the cis-region that had the strongest association with the eGene's expression and allocated these 10,911 unique SVs into six bins on the basis of causality score quantiles, with the least-causal bin containing the $50 \%$ of SVs with the lowest scores. Next, we measured the enrichment of SVs in each causality bin at a diverse set of genomic annotations and in the core 15 chromatin segmentation states from the Roadmap Epigenomics Project using a permutation test based on shuffled genomic positions (see Methods) (Supplemental Figs. S3, S4). SVs in the most causal quantiles were strongly enriched in the exons of their associated eGenes, which is expected and confirms that our causality score is informative. We also observed an enrichment of causal SVs in the $10-\mathrm{kb}$ regions upstream of the TSS and downstream from the 3' UTR of the associated eGene. Additionally, there is a small enrichment of the causal SVs in segmental duplications, which is likely driven by large mCNVs at multicopy genes. However, predicted causal SVs were not enriched in any other genomic features tested, which suggests that although eSVs are generally found relatively close to their eGenes, they may be altering expression through diverse mechanisms, and our study is underpowered to identify enrichments in specific regulatory element classes. Alternatively, existing annotations may be insufficiently informative to detect functional enrichments for the variants and tissues analyzed here.

The number and diversity of tissues with available expression data allow us to evaluate the tissue specificity of eQTLs. We hypothesized that SVs might have more ubiquitous effects on gene expression than point variants owing to constitutively acting dosage changes or owing to complete deletion or duplication of regulatory elements rather than more subtle effects, for example, on transcription factor binding. To allow for facile comparisons between variant types, we limited this analysis to variant-gene pairs with a significant association in our eQTL analysis for which expression data were available across all 48 tissues. We used METASOFT (Han and Eskin 2011) to evaluate eQTL activity across all tissues and limited this analysis to eQTLs for which active $(m>0.9)$ or inactive $(m<0.1)$ status could be determined in at least 43 tissues. We found that coding SV-eQTLs are more constitutive than other eQTL classes, showing activity across a larger proportion of tissues compared with SNVand indel-eQTLs (Fig. 1E). Whereas $92.16 \%$ of coding SV-eQTLs are constitutively active-defined here as active in $>75 \%$ of tissues with known status-only $74.12 \%$ of coding SNV- and indel-QTLs are constitutive. However, the result at noncoding eQTLs is less clear: $74.86 \%$ of noncoding SV-eQTLs are constitutively active as defined above, and $74.12 \%$ of noncoding SNV- and indel-eQTLs are constitutive, which suggests that there are not significant differences between these variant categories. However, when we examine noncoding eQTLs that are active in $100 \%$ of tissues with known activity, $44.44 \%$ of noncoding SV-eQTLs are active in all known tissues compared with $26.23 \%$ of noncoding SNV- and indel-eQTLs (Supplemental Fig. S5). Overall, this analysis shows that coding SVs typically impact expression across many tissues, whereas smaller and noncoding variants tend to affect gene expression on a more tissue-specific basis. In contrast to coding SVeQTLs, noncoding SV-eQTLs show similar patterns of tissue specificity to noncoding SNV- and indel-eQTLs, indicating that these variant types are likely to function through similar mechanisms. However, it is important to note that noncoding SV-eQTL activity 
could not be determined by METASOFT in many tissues (Supplemental Fig. S6), so it is possible that the true tissue specificity of noncoding SVs may differ from noncoding SNVs and indels. This appears to be the result of relatively large effect-size standard errors for SV-eQTLs that result from genotyping inaccuracies. Although METASOFT can determine cross-tissue eQTL activity when effect sizes are large despite large standard errors, as seen in coding SV-eQTLs, when effect sizes are small but effect size errors are large, the algorithm often cannot confidently judge activity (Supplemental Fig. S7).

\section{Effects of rare SVs}

Rare SVs are enriched near genes with highly aberrant expression (Chiang et al. 2017) and are more likely to have large effect sizes compared with other variant types (Li et al. 2017). To assess the effects of rare SVs on gene expression, we identified genes in which individuals displayed highly aberrant gene expression levels compared with the data set as a whole. We limited this analysis to the 513 individuals of European descent to reduce the effects of population stratification and limited our analyses to the 47 tissues for which data were available for at least 70 European individuals (Supplemental Table S1). We defined 26,289 autosomal multitissue gene expression outliers (median $|\mathrm{Z}| \geq 2$ across all tissues in an individual) and 173,061 autosomal "tissue-restricted" outliers with highly aberrant expression $(|Z| \geq 4)$ in two or more tissues in the same individual. Next, we identified 13,768 "singleton" SVs no larger than $1 \mathrm{Mb}$ in size that were positively genotyped in
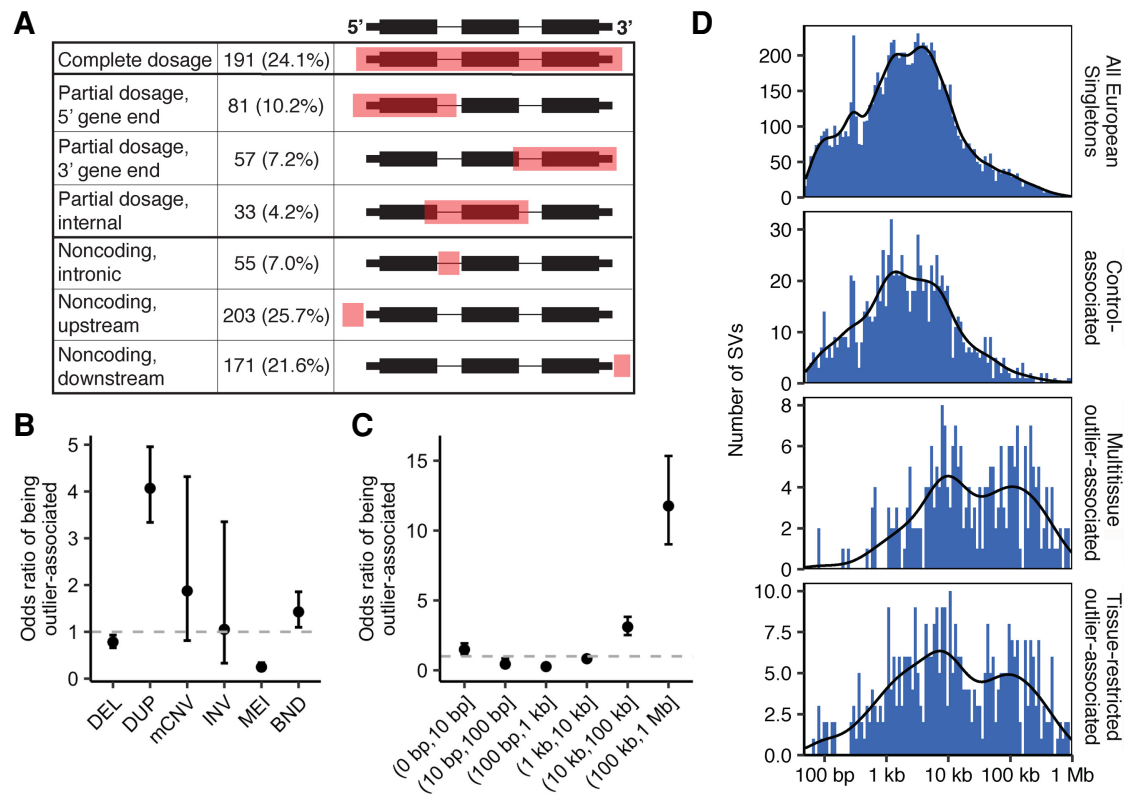

Figure 2. Features of outlier-associated SVs. $(A)$ Location of outlier-associated SVs relative to their associated outlier gene and the number of SV/outlier gene associations identified in each category. Percentages indicate the fraction of outlier/SV pairs found at each relative location compared with the total number of SV/outlier gene associations. Note that this definition allows one SV to be associated with multiple outlier genes, and thus, the SV is counted in multiple categories. Gene diagrams provide examples of possible SV location, shown in red, relative to the outlier gene. $(B, C)$ Odds ratio (OR) of being outlier-associated by SV type $(B)$ and SV size $(C)$ for the SV category of interest compared with all other SVs. Note that BNDs were excluded from the size OR calculations owing to their ambiguous nature and thus size. (D) Distribution of SV sizes for singleton SV $<1 \mathrm{Mb}$ identified in European individuals that were used in outlier analyses. Panels depict size distributions for all European-cohort singletons, control-associated singletons, multitissue outlier-associated singletons, and tissue-restricted outlier-associated singletons. one individual. These rare SVs are strongly enriched within the gene body and flanking sequence of multitissue gene expression outliers compared with the null expectation in 1000 random peras of the outlier sample names, with enrichment decreasof rare SV in close proximity (14.1-fold enriched ; $95 \%$ confidence interval $[\mathrm{Cl}], 8.7-25.1 ; P<0.001)$ to work (Chiang et al. 2017), but the increased power in this study aling distanserve enrichment at greater distances as well. At flank(95\% CI 4.9-8.8; $P<0.001)$ of rare SVs around multitissue outliers, suggesting that rare SVs contribute to rare expression differences gene expression values can only decrease to zero, a conservative fimit such as the one used for tissue-restricted outliers faiting our exility to deters (Supplemental Fig. S9). However, these conservative outlier definitions, combined with the above enrichment results, provide condence in the set of outlier-associated SVs.

of 539 unique outlier-associated (Fig. 2A; Supplemental Table S4). A majority of these (62.34\%; $336 / 539$ ) are noncoding SVs that do not affect the coding sequence sumption that rare SVs typically act through gene dosage effects. In total, $16.92 \%(31,978 / 188,988)$ of expression outliers are associated with a rare SV, although outliers can also arise via nongenetic mechanisms. To evaluate the relative potential of different SV types or sizes to cause expression outliers, we calculated the odds ratio (OR) of being outlier-associated for the SV category of interest compared with all other SVs. Duplications (OR 4.07) and mCNVs (OR 1.87) are most likely to be associated with an expression outlier, MEIs are least likely (OR 0.25) (Fig. 2B), and larger SVs are more likely to be outlier-associated regardless of type (Fig. 2C). However, many outlier-associated SVs are smaller in size (Fig. 2D). For example, $13.33 \%(50 / 375)$ of SVs associated with tissue-restricted outliers are $<1 \mathrm{~kb}$, and nearly half $(49.33 \% ; 185 / 375)$ are $<10 \mathrm{~kb}$. Multitissue outlier-associated SVs tend to be slightly larger, with only $4.98 \%(12 / 241)<1 \mathrm{~kb}$ and $35.27 \%(85 /$ $241)<10 \mathrm{~kb}$. These results provide further evidence that rare SVs often affect gene expression through more complex mechanisms than large, dosage-altering events.

We next sought to determine if rare outlier-associated SVs are enriched in annotated genomic features. Although there was little signal in our enrichment analysis of common SVs, as described above, rare variants typically have larger effect sizes and are more likely to be deleterious. For this analysis, we defined a

\section{Genome Research}

www.genome.org 
set of "control" SVs that are located within or near genes but do not show expression effects. We identified 1405 singleton SVs (1327 noncoding) located within $50 \mathrm{~kb}$ of autosomal genes that showed consistent expression levels (|Z| $<1)$ across all tissues in an individual. Although this is not an ideal set of control SVs considering that some may in fact alter gene expression in tissues or at developmental time points for which expression was not measured, it is nonetheless a relatively conservative set of likely-nonfunctional SVs that can be used for comparison to outlier-associated SVs. We examined the overlap of both outlier- and control-associated noncoding SVs with annotated genomic features and with segmentation states from the Roadmap Epigenomics Project core 15state model (Fig. 3A). We observed significant enrichment of outlier-associated SVs in 5 of the 34 evaluated features and chromatin states (Fisher's exact test; Bonferroni $P<0.05$ ). Most of these significant associations are in Roadmap Epigenomics Project segmentation states in close proximity to transcribed genes, including transcription at the $5^{\prime}$ and $3^{\prime}$ end of genes showing both promoter and enhancer signatures, active TSSs, and regions flanking active TSSs. We also observed significant enrichment in the Roadmap Epigenomics Project segmentation state associated with zinc finger protein genes and in enhancer annotations from GeneHancer. It is important to note, however, that the number of overlaps observed in this analysis is small and that increased power might change these results. Thus, although rare SVs appear to have large effects on gene expression, most existing functional annotations are not very informative. Consistent with this, the distribution of SV impact scores (Ganel et al. 2017) is not significantly different between expression-altering SVs and control SVs (Supplemental Fig. S10).

We found that 115 (21.34\%) outlier-associated SVs are associated with more than one expression outlier and that eight (1.48\%) are associated with five to nine expression outliers, suggesting that many rare SVs may have regional effects. To evaluate these broader regional effects of rare expression-altering SVs, we relaxed the definition for aberrant expression to generate a set of "secondary" expression outliers in which the tissue-restricted ("primary") outlier absolute $Z$-score cutoff was reduced to three in at least two tissues. We found significantly more primary and secondary outliers within $1 \mathrm{Mb}$ of the 469 tissue-restricted outlier-associated SVs compared with the 1405 control-associated SVs and with a null distribution in which we randomly shuffled the sample names of outlier-associated SVs 1000 times and calculated the median number of associated outlier genes (Fig. 3B,C). This increase is especially pronounced for secondary outliers whose coding regions do not overlap with the associated SV. We observe that noncoding outlier-associated SVs are associated with an average of 1.44 primary outliers $(|\mathrm{Z}| \geq 4)$ compared with an average of 0.02 associated primary outliers surrounding the shuffled null SVs $(P$-value $=$ $2.78 \times 10^{-106}$; one-sided Mann-Whitney $U$ test). These differences remain for secondary outliers, with an average of 3.34 secondary outliers found in the expanded region surrounding noncoding outlier-associated SVs compared with an average of 0.54 secondary outliers for the shuffled null $\left(P\right.$-value $=4.94 \times 10^{-76}$; one-sided Mann-Whitney $U$ test). These results suggest that rare SVs have far-reaching effects on gene expression and that these effects are primarily driven by noncoding regulatory mechanisms rather than by changes to gene copy number.

\section{Discussion}

We have comprehensively mapped SVs from WGS data in 613 individuals from the GTEx data set and analyzed the impact of both common and rare SVs on human gene expression. Our findings confirm results from previous analyses that SVs make an outsized contribution to common gene expression changes compared with their abundance in the genome and play an important role in rare gene expression differences (Chiang et al. 2017). A novel aspect of this study is the inclusion of a comprehensive set of MEI insertions, including those present in the GTEx samples but not the reference genome. We observed that MEIs do not play an especially important role in determining gene expression differences. In contrast, we found that mCNVs play an extremely impactful role, being 45-fold enriched among eQTL lead markers compared with their abundance in the genome and being more likely to be associated with gene expression outliers $(\mathrm{OR}=1.88)$. mCNVs were found to give rise to most human variation in gene dosage (Handsaker et al. 2015), but our findings indicate that noncoding functional mCNVs are also abundant in the human genome. 
One of the major motivators for studies such as this one is to understand the role of genetic variation in affecting gene transcription. Expression-altering SVs were not well correlated with any specific functional annotations other than proximity to genes, and thus, existing annotations are unlikely to be informative for modeling functional variant effects. This may simply be owing to a lack of power given that SVs are such a diverse class of variants that can affect large genomic segments and have the potential to affect gene expression through diverse mechanisms, and our sample size is limited to 11,026 common SVs and 539 rare SVs predicted to be functional. Alternatively, the annotations currently available may be inadequate.

Nonetheless, it is clear that SVs have broad regional impacts on human gene expression, with individual variants frequently affecting multiple genes. These effects are not driven by large CNVs that alter the dosage of multiple coding sequences, as one might naively expect, but are most commonly observed for noncoding variants: Common noncoding eSVs affect an average of 1.50 unique genes, and rare noncoding SVs are associated with an average of 1.44 primary expression outlier genes. This observation suggests a mechanism by which rare noncoding SVs may be especially deleterious, and may help explain why prior work has estimated that a large number of rare noncoding deletions - an average of 19.1 per individual - appear to be under strong purifying selection (Abel et al. 2020). Furthermore, the burden of de novo CNVs has been associated with autism spectrum disorder, including for noncoding variants (Turner et al. 2017; Turner and Eichler 2019). Our results provide a mechanism through which individual noncoding SVs can have strong and potentially pleiotropic effects and thus a higher potential to contribute to disease.

Although this study represents the most comprehensive analysis of the impact of SVs on human gene expression to date, our call set is missing some of the most repetitive classes of SV, such as short tandem repeats. As long-read sequencing and variant calling methods improve, we will be able to gain additional insights into repetitive variants in the most complex regions of the genome. Despite the limitations of short-read sequencing data, this study shows the importance of comprehensive variant detection when evaluating genomic variants that contribute to gene expression and disease. SVs have a disproportionately large effect on common and rare gene expression changes and often affect multiple genes. Our findings reinforce the importance of comprehensive variant detection in the design of future trait mapping studies.

\section{Methods}

\section{Call set generation}

We obtained 613 WGS BAM files from the GTEx v7 release (NCBI database of Genotypes and Phenotypes [dbGaP; https://www.ncbi .nlm.nih.gov/gap/] accession phs000424.v7.p2, accessed June 1, 2016). These data were aligned to GRCh 37 , and we did not realign to GRCh38 for this analysis to allow for comparison between SVs and the SNV and indel data available for this GTEx release. Our use of GRCh37 rather than the newer GRCh38 will not significantly affect the results or conclusions of this study, considering that the vast majority of loci have similar sequence content and structure; however, we note that CNV calls at specific structurally complex loci can vary somewhat between references. SV calls were generated using both the SpeedSeq v0.1.1 pipeline (Chiang et al. 2015), which performs sample-level breakpoint detection via LUMPY v.0.2.13 (Layer et al. 2014) followed by population-scale merging and genotyping of SV calls via svtools v0.3.1 (Larson et al. 2019), and the GenomeSTRiP v2.00.1636 read-depth analysis pipeline (Handsaker et al. 2011), as described in our preliminary GTEx study (Chiang et al. 2017). The GenomeSTRiP false-discovery rate (FDR) was evaluated based on available Illumina Human Omni 5M gene expression array data $(n=161)$ using the GenomeSTRiP IntensityRankSumAnnotator. We limited GSCNQUAL to one or more for GenomeSTRiP deletions and to eight or more for multiallelic CNVs, corresponding to an FDR of $10 \%$. The GSCNQUAL cutoff for GenomeSTRiP duplications was set at 17 or more, the point at which the FDR plateaued at $15.1 \%$ and did not fluctuate more than $\pm 1 \%$ for over 50 steps of increasing GSCNQUAL score. Redundant LUMPY and GenomeSTRiP calls were merged as previously described (Chiang et al. 2017). Additionally, we ran MELT v2.1.4 using MELT-SPLIT to identify Alu, SVA, and LINE-1 insertions into the test genomes (Gardner et al. 2017). We retained MELT calls categorized as "PASS" in the VCF info field that had an ASSESS score of three or more and SR count of three or more. Genome Analysis Toolkit (GATK) HaplotypeCaller v3.4 (McKenna et al. 2010) SNV and indel calls were obtained from the GTEx Consortium (dbGaP accession phs000424.v7.p2, accessed June 1, 2016). We use allele balance instead of genotype for the analyses described in this paper because it is tolerant to alignment inefficiencies for the alternate SV allele. For MEIs identified by MELT, we converted generated genotypes $(0 / 0$, $0 / 1,1 / 1)$ to integer values $(0,1,2)$ that were used as a proxy for allele balance to allow for comparable analyses on these variants.

\section{Common eQTL mapping}

We mapped cis-eQTLs in each of the 48 tissues for which both WGS data and RNA-seq data were available in 70 or more individuals. Available tissues and those used in each analysis are listed in Supplemental Table S1. We refer to EBV-transformed lymphocytes and transformed fibroblasts as tissue types throughout this study for convenience. Biospecimen collection, RNA-seq data alignment, RPKM calculations, and data normalization were previously described (Lappalainen et al. 2013; Chiang et al. 2017).

We selected common genetic markers, defined as having $\mathrm{MAF} \geq 0.01$, for eQTL mapping. We performed a joint cis-eQTL analysis that included 26,409 common SVs, as well as 9,609,545 common SNVs and 818,401 common indels detected using GATK, to allow for a fair comparison of the contribution of different variant types. We used FastQTL v2.184 (Ongen et al. 2016) to perform cis-eQTL mapping, customized to accommodate the unique architecture of SVs (Chiang et al. 2017), using a cis window of $1 \mathrm{Mb}$ on either side of the TSSs of autosomal and $\mathrm{X}$ Chromosome genes with a permutation analysis to identify the most significant marker for each gene. For each tissue, we applied the same covariates described by Chiang et al. (2017). We corrected for multiple testing at the gene level using the BenjaminiHochberg method with a 10\% FDR.

To evaluate the quality of our MEI-eQTLs, we performed a size-matched analysis by randomly selecting 286 LUMPY deletions to match the size distribution, measured in 50-bp bins, of the 6458 MEIs included in this study. We then calculated the percentage of selected LUMPY deletions that cause an eQTL compared with the number of MEI-eQTLs. We only used deletions detected by LUMPY for this analysis because they are mapped to high resolution and because we can be confident of their size. However, there are few deletions as small as MEIs, and thus, only a small subset of deletions was selected in order to match the two size distributions.

We calculated $\mathrm{R}^{2}$ between each $\mathrm{SV}$ and its best tagging SNV using the GenomeSTRiP TagVariantsAnnotator (Handsaker et al. 2015).

\section{Genome Research}

www.genome.org 


\section{Feature enrichment}

To evaluate whether SVs that cause common gene expression changes are enriched in particular genomic features, we calculated a previously described causality score (Chiang et al. 2017) generated by taking the product of the SV heritability fraction obtained from GCTA (Yang et al. 2011) and the causal probability generated by CAVIAR (2014) for the strongest-associated SV within the cis region of each eGene. No associated SVs were identified in 199 eQTLs owing to the subset of samples with available data in the relevant tissue and thus were not included in enrichment analyses. GCTA heritability estimates could not be calculated for a small number of eQTLs $(6146 / 299,187)$ owing to nonpositive definite matrices, likely resulting from small sample sizes, and these loci were excluded from feature enrichment analyses. For SVs that were associated with multiple eQTLs or the same eQTL in multiple tissues, we selected the eQTL (tissue/gene pair) for which the SV had the highest causality score. SVs were allocated into bins based on causality score quantiles, with the first bin consisting of SVs in the bottom $50 \%$ of causality scores and the other five consisting of deciles of the top $50 \%$ of scores.

Next, we counted the number of SVs in each bin that intersected with various genomic annotations. We allowed $1 \mathrm{~kb}$ of flanking distance surrounding all annotations with the following exceptions: GENCODE exons, no flanking distance; proximity to TSS and 3' gene end, $10 \mathrm{~kb}$ of directional flanking distance; topologically associated domain boundaries, $5 \mathrm{~kb}$ of flaking distance; and Roadmap Epigenomics segmentation states, no flanking distance. SVs associated with multiple eGenes were considered to touch an eGene if they overlapped with the exons of any associated gene. SVs that touched an exon of an associated eGene were excluded from all feature enrichment analyses except for the enrichment of affected eGene exons. To generate a shuffled null for comparison, SVs within each causality bin were shuffled with BEDTools v2.23.0 (Quinlan and Hall 2010) into nongapped regions of the genome within $1 \mathrm{Mb}$ of the TSS of a gene. We did not allow shuffled SVs to intersect any exons of their new eGene for all feature enrichment analyses, except for the enrichment of affected eGene exons. We calculated the fold enrichment of the number of SVs that intersect with each genomic feature compared with the median number of intersections observed for 100 randomly shuffled sets within each causality bin. These shuffled sets were also used to empirically derive the $95 \%$ confidence intervals.

The flanking distances indicated above were included to be consistent with our prior publication (Chiang et al. 2017), in which we observed that the enrichments were notably stronger for certain annotations with imprecise boundaries when we included the padding. However, we repeated the enrichment analysis for this study without the padding approach and found that the results look very similar to the results with padding and do not alter our conclusions (Supplemental Fig. S11).

Regions $10 \mathrm{~kb}$ upstream of TSSs and downstream from 3' gene ends were defined based on GENCODE v19 gene positions. DNase I hypersensitive regions and enhancer regions with a minimum support of two were obtained from the Dragon ENhancers database (DENdb) (Ashoor et al. 2015). We downloaded FunSeq 2.1.0 (Fu et al. 2014) regions and topologically associated domain boundaries from human embryonic stem cells from investigator websites (http://archive.gersteinlab.org/funseq2.1.0_data/ and http://compbio.med.harvard.edu/modencode/webpage/hic/ hESC_domains_hg19.bed). GeneHancer (Fishilevich et al. 2017) enhancer regions for b38 were downloaded from the UCSC Genome Browser (Kent et al. 2002) and lifted over to b37 using CrossMap v0.2.6 (Zhao et al. 2014). Regions defined by the ENCODE Project (The ENCODE Project Consortium 2012) were downloaded from the UCSC Genome Browser. To evaluate the intersection with the chromatin segmentation state annotations from the Roadmap Epigenomics Project (Roadmap Epigenomics Consortium et al. 2015), we downloaded the core 15-state model annotations for all 127 available epigenomes (https://egg2 .wustl.edu/roadmap/data/byFileType/chromhmmSegmentations/ ChmmModels/coreMarks/jointModel/final). We used BEDTools multiIntersectBed (Quinlan and Hall 2010) to identify genomic intervals where each of the 15 annotations is found in at least 10 of the 127 available epigenomes, and we used these collapsed regions as the annotation intervals for SV intersections.

\section{eQTL tissue specificity}

We selected significant gene-variant pairs identified in eQTL mapping with available expression data available across all 48 tissues in which the eQTL analyses were performed. These pairs were only required to have a significant eQTL in one tissue. We used METASOFT v2.0.0 (Han and Eskin 2011) to perform a meta-analysis of the selected eQTL effect sizes and their standard errors across all 48 tissues. METASOFT uses a mixed effects model (RE2) to generate a posterior probability that an effect exists in each tissue $(\mathrm{m}$ value) (Han and Eskin 2012). To allow computational feasibility with the relatively large number of tissues sampled, the Markov chain Monte Carlo (MCMC) method was used to approximate these values. The $m$-values generated indicate whether a tested eQTL is active $(m>0.9)$, is inactive $(m<0.1)$, or has ambiguous activity $(0.1 \leq m \leq 0.9)$. Only eQTLs with at least 43 tissues having known (active or inactive) activity were included in analyses. eQTLs with active status in at least 75\% of tissues with known activity were defined as "constitutively active."

\section{Identification of expression outliers}

We limited outlier analyses to the 513 European individuals, the largest subpopulation in the cohort, who had available WGS data. We performed Z-transformation of PEER-corrected expression values without quantile normalization across the 47 tissues for which RNA-seq data were available from the GTEx Consortium for at least 70 European individuals (Supplemental Table S1). We defined two sets of gene expression outliers (gene/ sample pairs) among these individuals: "multitissue" expression outliers in which an individual's absolute median $Z$-score of a gene's expression across all available tissues was two or more, as previously described (Chiang et al. 2017), and "tissue-restricted" outliers in which an individual's absolute $Z$-score for a gene's expression was four or more in at least two different tissues. The two-tissue requirement was necessary to eliminate false-positive expression outliers resulting from individual tissues with systematically aberrant gene expression profiles for an individual. Additionally, we defined a set of control gene/sample pairs in which an individual's absolute $Z$-score of a gene's expression was less than one across all tissues for which RNA-seq data were available. For all definitions, we limited to gene/sample pairs with data available in at least five tissues. We removed one individual (GTEX-14753) from this analysis owing to an excessive number of expression outliers.

\section{Rare variant association with expression outliers}

We identified 13,769 SVs that were positively genotyped in no more than one individual in the European cohort. Because large rare SVs tend to affect gene expression through dosage changes, we removed 12 variants $>1 \mathrm{Mb}$ in size from this analysis. We calculated the enrichment of singleton SVs overlapping with multitissue outlier transcripts and the flanking $5-\mathrm{kb}$ sequence by 
randomly shuffling the outlier individual names 1000 times to determine the median number of times a rare variant randomly co-occurred with an outlier, as described previously (Chiang et al. 2017). We also performed the reciprocal analysis, counting the number of outliers that co-occurred within $5 \mathrm{~kb}$ of a rare SV. We repeated these calculations for increased outlier-flanking regions of $10 \mathrm{~kb}, 25 \mathrm{~kb}, 50 \mathrm{~kb}$, and $100 \mathrm{~kb}$. We calculated the OR of being outlier-associated by dividing the ratio of outlier-associated SVs to non-outlier-associated SVs in a category of interest (SV type or size) by the ratio of outlier-associated SVs to non-outlier-associated SVs for all SVs not included in the category.

\section{Feature enrichment for outlier-associated SVs}

We performed intersections between the 373 noncoding outlierassociated SVs and the same genomic features and chromatin segmentation states evaluated for eSVs. The above intersections were repeated for the 1327 noncoding control-associated SVs. We calculated the fold enrichment of outlier-associated SVs in each feature compared with control-associated SVs, and determined significant enrichments using a Fisher's exact test with Bonferroni correction for multiple testing.

\section{Regional effects of rare SVs}

To evaluate the broader regional effects of rare, gene expression-altering SVs, we counted the number of tissue-restricted outlier genes, referred to as "primary" outliers, located in the spanning region and $1 \mathrm{Mb}$ of flanking sequence both upstream of and downstream from the 469 SVs previously identified as being associated with a tissue-restricted expression outlier. We repeated this analysis with a relaxed definition of tissue-restricted expression outliers, referred to as "secondary" outliers, in which the absolute $Z$-score cutoff was reduced from $|Z| \geq 4$ to $|Z| \geq 3$. We compared the number of primary and secondary outliers found in the expanded region surrounding outlier-associated SVs to the expanded region surrounding the 1405 control-associated SVs. Finally, because the controls defined above do not represent a null expectation, we performed 1000 random permutations of the outlier-associated SV sample names and calculated the median number of associated primary and secondary outliers for each SV in order to determine how frequently rare expression-altering SVs co-occurred with primary and secondary outliers in random individuals.

\section{Data access}

The SV genotype data generated in this study have been submitted to AnVIL and can be downloaded from the GTEx v7 workspace (https://app.terra.bio/\#workspaces/anvil-datastorage/

AnVIL_GTEx_V7_hg19). A VCF file containing all SV calls and genotypes, including non-PASS variants that failed quality filters (GTEx_v7.sv.low_pass.vcf), and an accompanying README file (GTEx_v7_SV_README.txt) are available in this workspace. A Terra account and dbGaP access to GTEx (accession number phs000424) are required.

\section{Competing interest statement}

The authors declare no competing interests.

\section{Acknowledgments}

We thank E.J. Gardner for advice on MELT and R.E. Handsaker for advice on GenomeSTRiP. This work was supported by a Mr. and Mrs. Spencer T. Olin Fellowship for Women in Graduate Study
(A.J.S.) and by the National Institutes of Health/National Human Genome Research Institute UM1 HG008853 (I.M.H.).

\section{References}

Abel HJ, Larson DE, Rieger AA, Chiang C, Das I, Kanchi KL, Layer RM, Neale BM, Salerno WJ, Reeves C, et al. 2020. Mapping and characterization of structural variation in 17,795 human genomes. Nature 583: 83-89. doi:10.1038/s41586-020-2371-0

Ashoor H, Kleftogiannis D, Radovanovic A, Bajic VB. 2015. DENdb: database of integrated human enhancers. Database 2015: bav085. doi:10 $.1093 /$ database/bav085

Brandler WM, Antaki D, Gujral M, Kleiber ML, Whitney J, Maile MS, Hong O, Chapman TR, Tan S, Tandon P, et al. 2018. Paternally inherited cisregulatory structural variants are associated with autism. Science 360: 327-331. doi:10.1126/science.aan2261

Chiang C, Layer RM, Faust GG, Lindberg MR, Rose DB, Garrison EP, Marth GT, Quinlan AR, Hall IM. 2015. SpeedSeq: ultra-fast personal genome analysis and interpretation. Nat Methods 12: 966-968. doi:10.1038/ nmeth.3505

Chiang C, Scott AJ, Davis JR, Tsang EK, Li X, Kim Y, Hadzic T, Damani FN, Ganel L, GTEx Consortium, et al. 2017. The impact of structural variation on human gene expression. Nat Genet 49: 692-699. doi:10.1038/ ng.3834

Chuong EB, Elde NC, Feschotte C. 2017. Regulatory activities of transposable elements: from conflicts to benefits. Nat Rev Genet 18: 71-86. doi:10.1038/nrg.2016.139

Collins RL, Brand H, Karczewski KJ, Zhao X, Alföldi J, Francioli LC, Khera AV, Lowther C, Gauthier LD, Wang H, et al. 2020. A structural variation reference for medical and population genetics. Nature 581: 444-451. doi:10.1038/s41586-020-2287-8

Ebert P, Audano PA, Zhu Q, Rodriguez-Martin B, Porubsky D, Bonder MJ, Sulovari A, Ebler J, Zhou W, Serra Mari R, et al. 2021. Haplotype-resolved diverse human genomes and integrated analysis of structural variation. Science 372: eabf7117. doi:10.1126/science.abf7117

The ENCODE Project Consortium. 2012. An integrated encyclopedia of DNA elements in the human genome. Nature 489: 57-74. doi:10 $1038 /$ nature 11247

Fishilevich S, Nudel R, Rappaport N, Hadar R, Plaschkes I, Iny Stein T, Rosen N, Kohn A, Twik M, Safran M, et al. 2017. GeneHancer: genome-wide integration of enhancers and target genes in GeneCards. Database 2017: bax028. doi:10.1093/database/bax028

Fu Y, Liu Z, Lou S, Bedford J, Mu XJ, Yip KY, Khurana E, Gerstein M. 2014 FunSeq2: a framework for prioritizing noncoding regulatory variants in cancer. Genome Biol 15: 480. doi:10.1186/s13059-014-0480-5

Ganel L, Abel HJ, FinMetSeq Consortium, Hall IM. 2017. SVScore: an impact prediction tool for structural variation. Bioinformatics 33: 1083-1085. doi:10.1093/bioinformatics/btw789

Gardner EJ, Lam VK, Harris DN, Chuang NT, Scott EC, Pittard WS, Mills RE, The 1000 Genomes Project Consortium, Devine SE. 2017. The mobile element locator tool (MELT): population-scale mobile element discovery and biology. Genome Res 27: 1916-1929. doi:10.1101/gr.218032 .116

Han B, Eskin E. 2011. Random-effects model aimed at discovering associations in meta-analysis of genome-wide association studies. Am J Hum Genet 88: 586-598. doi:10.1016/j.ajhg.2011.04.014

Han B, Eskin E. 2012. Interpreting meta-analyses of genome-wide association studies. PLoS Genet 8: e1002555. doi:10.1371/journal.pgen .1002555

Han L, Zhao X, Benton ML, Perumal T, Collins RL, Hoffman GE, Johnson JS, Sloofman L, Wang HZ, Stone MR, et al. 2020. Functional annotation of rare structural variation in the human brain. Nat Commun 11: 2990 . doi:10.1038/s41467-020-16736-1

Handsaker RE, Korn JM, Nemesh J, McCarroll SA. 2011. Discovery and genotyping of genome structural polymorphism by sequencing on a population scale. Nat Genet 43: 269-276. doi:10.1038/ng.768

Handsaker RE, Van Doren V, Berman JR, Genovese G, Kashin S, Boettger LM, McCarroll SA. 2015. Large multiallelic copy number variations in humans. Nat Genet 47: 296-303. doi:10.1038/ng.3200

Hormozdiari F, Kostem E, Kang EY, Pasaniuc B, Eskin E. 2014. Identifying causal variants at loci with multiple signals of association. Genetics 198: 497-508. doi:10.1534/genetics.114.167908

International Schizophrenia Consortium. 2008. Rare chromosomal deletions and duplications increase risk of schizophrenia. Nature 455: 237-241. doi:10.1038/nature07239

Jakubosky D, D'Antonio M, Bonder MJ, Smail C, Donovan MKR, Young Greenwald WW, Matsui H, i2QTL Consortium, D'AntonioChronowska A, Stegle O, et al. 2020. Properties of structural variants

\section{Genome Research}

www.genome.org 
and short tandem repeats associated with gene expression and complex traits. Nat Commun 11: 2927. doi:10.1038/s41467-020-16482-4

Kent WJ, Sugnet CW, Furey TS, Roskin KM, Pringle TH, Zahler AM, Haussler D. 2002. The human genome browser at UCSC. Genome Res 12: 9961006. doi:10.1101/gr.229102

Lappalainen T, Sammeth M, Friedländer MR, 't Hoen PA, Monlong J, Rivas MA, Gonzàlez-Porta M, Kurbatova N, Griebel T, Ferreira PG, et al. 2013. Transcriptome and genome sequencing uncovers functional variation in humans. Nature 501: 506-511. doi:10.1038/nature12531

Larson DE, Abel HJ, Chiang C, Badve A, Das I, Eldred JM, Layer RM, Hall IM. 2019. svtools: population-scale analysis of structural variation. Bioinformatics 35: 4782-4787. doi:10.1093/bioinformatics/btz492

Layer RM, Chiang C, Quinlan AR, Hall IM. 2014. LUMPY: a probabilistic framework for structural variant discovery. Genome Biol 15: R84. doi:10.1186/gb-2014-15-6-r84

Li X, Kim Y, Tsang EK, Davis JR, Damani FN, Chiang C, Hess GT, Zappala Z, Strober BJ, Scott AJ, et al. 2017. The impact of rare variation on gene expression across tissues. Nature 550: 239-243. doi:10.1038/nature24267

Marshall CR, Howrigan DP, Merico D, Thiruvahindrapuram B, Wu W, Greer DS, Antaki D, Shetty A, Holmans PA, Pinto D, et al. 2017. Contribution of copy number variants to schizophrenia from a genome-wide study of 41,321 subjects. Nat Genet 49: 27-35. doi:10.1038/ng.3725

McCarthy SE, Makarov V, Kirov G, Addington AM, McClellan J, Yoon S, Perkins DO, Dickel DE, Kusenda M, Krastoshevsky O, et al. 2009. Microduplications of $16 \mathrm{p} 11.2$ are associated with schizophrenia. Nat Genet 41: $1223-1227$. doi:10.1038/ng.474

McKenna A, Hanna M, Banks E, Sivachenko A, Cibulskis K, Kernytsky A, Garimella K, Altshuler D, Gabriel S, Daly M, et al. 2010. The Genome Analysis Toolkit: a MapReduce framework for analyzing next-generation DNA sequencing data. Genome Res 20: 1297-1303. doi:10.1101/ gr.107524.110

Ongen H, Buil A, Brown AA, Dermitzakis ET, Delaneau O. 2016. Fast and efficient QTL mapper for thousands of molecular phenotypes. Bioinformatics 32: 1479-1485. doi:10.1093/bioinformatics/btv722

Payer LM, Burns KH. 2019. Transposable elements in human genetic disease. Nat Rev Genet 20: 760-772. doi:10.1038/s41576-019-0165-8

Quinlan AR, Hall IM. 2010. BEDTools: a flexible suite of utilities for comparing genomic features. Bioinformatics 26: 841-842. doi:10.1093/bioinfor matics/btq033

Roadmap Epigenomics Consortium, Kundaje A, Meuleman W, Ernst J, Bilenky M, Yen A, Heravi-Moussavi A, Kheradpour P, Zhang Z, Wang
J, Ziller MJ, et al. 2015. Integrative analysis of 111 reference human epigenomes. Nature 518: 317-330. doi:10.1038/nature14248

Sebat J, Lakshmi B, Malhotra D, Troge J, Lese-Martin C, Walsh T, Yamrom B, Yoon S, Krasnitz A, Kendall J, et al. 2007. Strong association of de novo copy number mutations with autism. Science 316: 445-449. doi:10 .1126/science.1138659

Stranger BE, Forrest MS, Dunning M, Ingle CE, Beazley C, Thorne N, Redon R, Bird CP, de Grassi A, Lee C, et al. 2007. Relative impact of nucleotide and copy number variation on gene expression phenotypes. Science 315: $848-853$. doi: $10.1126 /$ science. 1136678

Sudmant PH, Rausch T, Gardner EJ, Handsaker RE, Abyzov A, Huddleston J, Zhang Y, Ye K, Jun G, Hsi-Yang Fritz M, et al. 2015. An integrated map of structural variation in 2,504 human genomes. Nature 526: 75-81. doi:10.1038/nature15394

Turner TN, Eichler EE. 2019. The role of de novo noncoding regulatory mutations in neurodevelopmental disorders. Trends Neurosci 42: 115-127. doi:10.1016/j.tins.2018.11.002

Turner TN, Coe BP, Dickel DE, Hoekzema K, Nelson BJ, Zody MC Kronenberg ZN, Hormozdiari F, Raja A, Pennacchio LA, et al. 2017. Genomic patterns of de novo mutation in simplex autism. Cell 171: 710-722.e12. doi:10.1016/j.cell.2017.08.047

Walsh T, McClellan JM, McCarthy SE, Addington AM, Pierce SB, Cooper GM, Nord AS, Kusenda M, Malhotra D, Bhandari A, et al. 2008. Rare structural variants disrupt multiple genes in neurodevelopmental pathways in schizophrenia. Science 320: 539-543. doi:10.1126/science .1155174

Weiss LA, Shen Y, Korn JM, Arking DE, Miller DT, Fossdal R, Saemundsen E, Stefansson H, Ferreira MA, Green T, et al. 2008. Association between microdeletion and microduplication at 16p11.2 and autism. $N$ Engl J Med 358: 667-675. doi:10.1056/NEJMoa075974

Yang J, Lee SH, Goddard ME, Visscher PM. 2011. GCTA: a tool for genomewide complex trait analysis. Am J Hum Genet 88: 76-82. doi:10.1016/j ajhg.2010.11.011

Zhao H, Sun Z, Wang J, Huang H, Kocher J-P, Wang L. 2014. CrossMap: a versatile tool for coordinate conversion between genome assemblies. Bioinformatics 30: 1006-1007. doi:10.1093/bioinformatics/btt730

Received March 6, 2021; accepted in revised form September 14, 2021. 


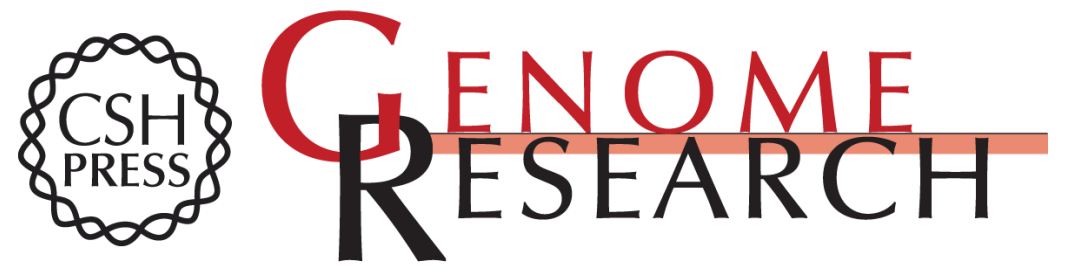

\section{Structural variants are a major source of gene expression differences in humans and often affect multiple nearby genes}

Alexandra J. Scott, Colby Chiang and Ira M. Hall

Genome Res. published online September 20, 2021

Access the most recent version at doi:10.1101/gr.275488.121

Supplemental Material

$\mathbf{P}<\mathbf{P} \quad$ Published online September 20, 2021 in advance of the print journal.

Open Access Freely available online through the Genome Research Open Access option.

Creative This article, published in Genome Research, is available under a Creative Commons

License

Email Alerting Service

http://genome.cshlp.org/content/suppl/2021/11/12/gr.275488.121.DC1

Commons License (Attribution 4.0 International), as described at

http://creativecommons.org/licenses/by/4.0/.

Receive free email alerts when new articles cite this article - sign up in the box at the top right corner of the article or click here.

\section{Affordable, Accurate Sequencing.}

To subscribe to Genome Research go to: https://genome.cshlp.org/subscriptions 